\title{
The Ebola virus model based on the cellular automata
}

\author{
Fei Lu \\ North China Electric Power University (Baoding), Baoding, 071000, China
}

Keywords: Ebola virus, cellular automata, randomly moving.

\begin{abstract}
The spreading of infectious diseases is a process of evolution with time going. In this paper we analyze the spreading rules of the infectious diseases and set up a model to simulate the dynamic spreading process based on the cellular automata model. Through the improved cellular automata model, this paper describes the developing process of the diseases in a population, predicts the infected state and finally provides an optimal decision for preventing and controlling the diseases.
\end{abstract}

\section{Introduction}

The existing data indicate Ebola virus quickly spreading in the Africa in a short time. At present Ebola virus have caused a number of people infected and dead from Aug 2014 when Ebola out broke in West Africa ${ }^{[1]}$. Thus this paper set up a mathematic model to describe the process of its spreading. This paper gets the states about the spreading of epidemics under single region restriction and multiple region restriction. The simulated result can well fit the real condition. Based on the model we analyze the infecting regularity in a population and predict the moment when the infected condition comes to a point that some controlling action should be taken.

\section{The epidemic model basing on the cellular automata [2]}

A cellular automaton is a commonly used method to describe the complicated phenomenon in reality. It is a dynamical system discrete in time, space and state so it can evolve to a complex state. Through adding some regulations set according to the rules of spreading to simulate the complicated process of spreading.

\section{The spreading model basing on the cellular automata}

\section{Cellular automata and the randomly moving cellular automata.}

Basing on the good ability of cellular automata to model complex problems, we set the evolution rules in the cellular automata to simulate the complicated epidemic spreading process. The improved cellular automata can be defined as:

$$
A=(L d, \bar{S}, N, f)
$$

In the formula: $A$ is the cellular system; $L d$ means $d$ dimensional space and $d$ is positive integer;

$\bar{S}$ is the discrete set about the state of cellules; $N$ means the state of a cellule's neighborhood group (including the central cellular); $f$ means a transfer function from $S_{N}$ to $\bar{S}$ The evolution rules can be expressed as :

$$
S_{i}^{t+1}=f\left(S_{j_{1}}^{t}, \ldots, S_{j_{N}}^{t}\right)
$$

$S_{i}^{t+1}$ is the state of cellular $i$ in $t+1$ moment, $\left\{S_{j_{1}}^{t}, \ldots, S_{j_{N}}^{t}\right\}$ is the set of all the neighborhood of $S_{i}^{t}$.

\section{Assumptions.}

According to the methods ${ }^{[3]}$ to analyze the epidemic diseases, we apply a randomly moving cellular automaton in 2 dimensions ${ }^{[4]}$ to set up the epidemic diseases model. Besides we add the terrain factors creatively and divide the cellular automata into multiple areas to research the spreading 
model more accurately. Finally compare the results simulated in multiple regions with that in single region. Every moment each cellular has 6 states possibly:

$S_{i, j}^{t}=0$ : a terrain; $S_{i, j}^{t}=1$ : a normal person easily infected; $S_{i, j}^{t}=2$ : a infected person in incubation; $S_{i, j}^{t}=3$ : sick; $S_{i, j}^{t}=4$ : a person in isolation; $S_{i, j}^{t}=5$ : dead;

1) The length of step to the cellular automata obeys POISSON distribution:

$D \sim P(5)$

2) The rate to the infected person in incubation diagnosed and isolated:

$P_{1}=0.3$

3) The rate to sick person in to be diagnosed and isolated:

$P_{2}=0.8$

4) The incubation period of infected person:

$T_{1} \sim N(11.5,5)$

5) The rate of infected persons in incubation to infect normal people:

$P_{3}=0.05$

6) The rate of sick people to infect normal people:

$P_{4}=0.2$

7) The rate of isolated people to be cured:

$P_{5}=0.9$

\section{The evolution rules in the cellular automata.}

Mesh the 2-D space to be simulated uniformly and set the characteristic parameters about the Ebola virus. Based on the reality, the initial state of the cellular system contains terrain factors and normal people and the ratio of the number of the 2 factors is 1:9. According to that ratio, randomly set the cellular initial state $S_{i, j}^{0}=0$ or $S_{i, j}^{0}=1$ and add several cellular in the state $S_{i, j}^{0}=3$ to the cellular system. Beginning at the moment 0 , scan all the cellular in the system and have random moving operation to the cellulas a time every evolution in the step set before. Finally update the state of all the cellulas following the rules below:

When $S_{i, j}^{t}=0$, it means this cellular is a terrain of which the state is unchangeable.

$S_{i, j}^{t+1}=S_{i, j}^{t}$

When $S_{i, j}^{t}=1$, it mean this cellular is a normal person and calculate the rate to be infected $P_{7}\left(S_{i, j}^{t}\right)$ :

$P_{6}\left(S_{i, j}^{t}\right)=1-\left(1-P_{3}\right)^{N_{1}}\left(1-P_{4}\right)^{N_{2}}$

In the formula: $N_{1}$ is the number of infected people in a $5 \times 5$ set around the cellular. $N_{2}$ is the number of sick person in a $5 \times 5$ set around it. Finally according to the $P_{7}\left(S_{i, j}^{t}\right)$, the state of the central cellular can be changed to $S_{i, j}^{t+1}=2$.

c) When $S_{i, j}^{t}=2$, it means this cellular is an infected person in incubation. Basing on the rate $P_{7}=P\left(T_{1} \leq T_{S}\left(S_{i, j}^{t+1}\right)\right)$, judge whether the state of this cellular should be changed to $S_{i, j}^{t+1}=3$. Basing on $P_{1}=0.3$, judge whether the state of this cellular should be changed to $S_{i, j}^{t+1}=4$.

d) When $S_{i, j}^{t}=3$, it means this cellular is a sick person. Basing on the rate ${ }_{P_{8}}{ }^{=} P\left(T_{2} \leq T_{s}\left(S_{i, j}^{t}\right)\right)$, judge whether the state of this cellular should be changed to $S_{i, j}^{t+1}=5$. Basing on the rate $p_{2}=0.8$, judge whether the state of this cellular should be changed to $S_{i, j}^{t+1}=4$.

e) When $S_{i, j}^{t}=4$, it means this cellular is a sick person isolated. Basing on the rate $P_{5}=0.9$, judge whether the state of this cellular should be change to $S_{i, j}^{t+1}=1$. 


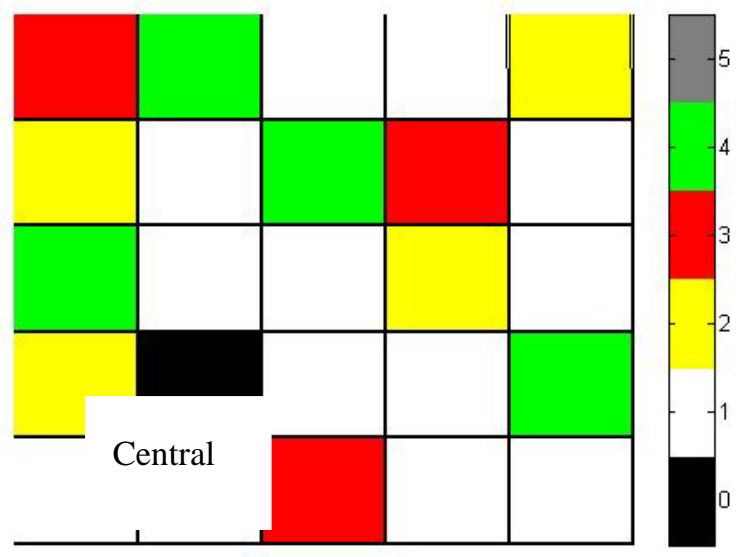

Figure 1 the diagram about the group around the central cellular

\section{Solution and Result.}

According to the characteristics of Ebola, this paper runs the improved cellular automata system to simulate the spreading process of Ebola. Ebola is a kind of virus with high fatality rate and highly infectious. The result is controllable but not periodic with the condition: isolation and medicine. The simulated results are as follows:

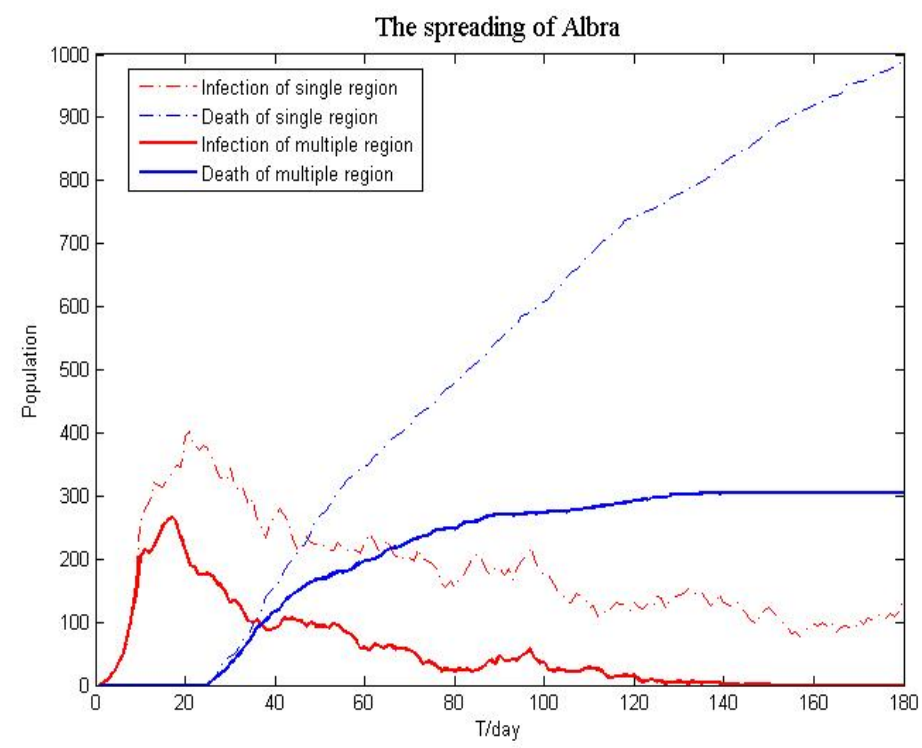

Figure 2 the number of person infected and dead in single region and multiple region

From tendency chart about the number of person infected and dead in single region, we can see that the number of infected people increase sharply with time passing by, which indicates Ebola has strong infecting ability without human intervention at the beginning. When the iteration step gets to 20 ( $20^{\text {th }}$ day), the number of infected people gets to the peak. After that peak, the number of infected people begins to decrease that tells medicines and isolation measurement comes into effect. From the tendency chart above, it's obvious to us that the tendency of the simulated results in the multiple region is similar to that tendency in the single region. That implies Ebola's spreading patterns are almost in the same way both in the single region and multiple regions. Multiple regions restriction has a better limitation

We can see that multiple region has a more stable trend when compared with a single region. Infected people in multiple region decrease by a reduction of $25 \%$, and the number of dead people tends to be a convergence in the later period. The results are consistent with the development of large flu in the actual situation, in which topographic factor plays a very important role. By the comparison between the simulated results in the two different regions, we can draw the conclusion that in the earlier stage of spreading, timely isolation measurement helps to control the spread of Ebola virus. 


\section{Summary}

Firstly, based on the existing cellular automaton model, we apply a randomly moving cellular automaton in 2 dimensions ${ }^{[6]}$ to set up the epidemic diseases model. Besides we add the terrain factors creatively and divide the cellular automata into multiple areas to research the spreading model more accurately. Through formulating cellular automaton evolution rules, this paper simulates the spread of epidemics. The simulated result can well fit the real condition. Secondly, considering the terrain, the original area is divided into $3 \times 3$ regions. Get the states about the spreading of epidemics under single region restriction and multiple region restriction. The result shows that multiple region restriction has a better resistance to the spreading of the epidemics, which indicates that it is helpful to the prevention of epidemic spreading to reinforce the area of denial.

\section{References}

[1]http://apps.who.int/ebola/en/ebola-situation-report/situation-reports/ebola-situation-report-4-febr uary-2015(2015.2.8)

[2] Von Neumann J.The general and logical theory of automata[C].In: Jeffries L A ed.Cerebral Mechanisms in Behavior-the Hixon Symposium, New York: Wiley, 1951

[3] Fumin Shen, Principles and methods of epidemiology [M]shanghai: Fudan University Press, 2001.

[4] Xin Yu, Xiaodong Duan, Xiangdong Liu, Based on cellular automaton epidemic model and its simulation [J]. Computer engineering and applications, 2005. 